\title{
On Certain Subclasses of Analytic Functions Involving Carlson-Shaffer Operator and Related to Lemniscate of Bernoulli
}

\author{
Jagannath Patel $^{1}$ and Ashok Kumar Sahoo ${ }^{2}$ \\ ${ }^{1}$ Department of Mathematics, Utkal University, Vani Vihar, Bhubaneswar 751004, India \\ ${ }^{2}$ Department of Mathematics, Veer Surendra Sai University of Technology, Sidhi Vihar, Burla 768018, India \\ Correspondence should be addressed to Jagannath Patel; jpatelmath@yahoo.co.in
}

Received 23 September 2014; Accepted 2 December 2014; Published 16 December 2014

Academic Editor: Alfonso Montes-Rodriguez

Copyright (C) 2014 J. Patel and A. K. Sahoo. This is an open access article distributed under the Creative Commons Attribution License, which permits unrestricted use, distribution, and reproduction in any medium, provided the original work is properly cited.

The object of the present investigation is to solve the Fekete-Szegö problem and determine the sharp upper bound to the second Hankel determinant for a new class $\mathscr{R}(a, c)$ of analytic functions involving the Carlson-Shaffer operator in the unit disk. We also obtain a sufficient condition for normalized analytic functions in the unit disk to be in this class.

\section{Introduction and Preliminaries}

Let $\mathscr{A}$ be the class of functions $f$ of the form

$$
f(z)=z+\sum_{k=2}^{\infty} a_{k} z^{k}
$$

which are analytic in the open unit disk $\mathbb{U}=\{z \in \mathbb{C}:|z|<1\}$.

A function $f \in \mathscr{A}$ is said to be starlike of order $\rho$, if

$$
\operatorname{Re}\left\{\frac{z f^{\prime}(z)}{f(z)}\right\}>\rho \quad(0 \leq \rho<1 ; z \in \mathbb{U}) .
$$

Similarly, a function $f \in \mathscr{A}$ is said to be convex of order $\rho$, if

$$
\operatorname{Re}\left\{1+\frac{z f^{\prime \prime}(z)}{f^{\prime}(z)}\right\}>\rho \quad(0 \leq \rho<1 ; z \in \mathbb{U}) .
$$

By usual notations, we write these classes of functions by $\mathcal{S}^{\star}(\rho)$ and $\mathscr{K}(\rho)$, respectively. We denote $\mathcal{S}^{\star}(0)=\mathcal{S}^{\star}$ and $\mathscr{K}(0)=\mathscr{K}$, the familiar subclasses of starlike, convex functions in $\mathbb{U}$.
Furthermore, let $\mathscr{P}$ denote the class of analytic functions $\phi$ normalized by

$$
\phi(z)=1+p_{1} z+p_{2} z^{2}+\cdots \quad(z \in \mathbb{U})
$$

such that $\operatorname{Re}\{\phi(z)\}>0$ in $\mathbb{U}$.

For functions $f$ and $g$, analytic in $\mathbb{U}$, we say that $f$ is subordinate to $g$, written as $f \prec g$ or $f(z) \prec g(z)(z \in \mathbb{U})$, if there exists a Schwarz function $\omega$, which (by definition) is analytic in $\mathbb{U}$ with $\omega(0)=0,|\omega(z)|<1$, and $f(z)=g(\omega(z))$, $z \in \mathbb{U}$. Furthermore, if the function $g$ is univalent in $\mathbb{U}$, then we have the following equivalence relation (cf., e.g., [1]; see also [2]):

$$
f(z) \prec g(z) \Longleftrightarrow f(0)=g(0), \quad f(\mathbb{U}) \subset g(\mathbb{U}) .
$$

For functions $f_{j}(z)=\sum_{k=0}^{\infty} a_{k, j} z^{k}(j=1,2)$ analytic in $\mathbb{U}$, we define the Hadamard product (or convolution) of $f_{1}$ and $f_{2}$ by

$$
\left(f_{1} \star f_{2}\right)(z)=\sum_{k=0}^{\infty} a_{k, 1} a_{k, 2} z^{k}=\left(f_{2} \star f_{1}\right)(z) \quad(z \in \mathbb{U}) .
$$

Note that $\left(f_{1} \star f_{2}\right)$ is also analytic in $\mathbb{U}$. 
Carlson and Shaffer [3] defined the linear operator $\mathscr{L}(a, c): \mathscr{A} \rightarrow \mathscr{A}$ in terms of the incomplete beta function $\varphi$ by

$$
\mathscr{L}(a, c) f(z)=\varphi(a, c ; z) \star f(z) \quad(f \in \mathscr{A} ; z \in \mathbb{U}),
$$

where

$$
\begin{aligned}
& \varphi(a, c ; z)=\sum_{k=0}^{\infty} \frac{(a)_{k}}{(c)_{k}} z^{k+1} \\
& \left(a \in \mathbb{C}, c \in \mathbb{C} \backslash \mathbb{Z}_{0}^{-}, \mathbb{Z}_{0}^{-}=\{\ldots,-2,-1,0\} ; z \in \mathbb{U}\right)
\end{aligned}
$$

and $(\varkappa)_{n}$ denotes the Pochhammer symbol (or shifted factorial) given, in terms of the Gamma function $\Gamma$, by

$$
\begin{aligned}
(\varkappa)_{n} & =\frac{\Gamma(\varkappa+n)}{\Gamma(\varkappa)} \\
& = \begin{cases}\varkappa(\varkappa+1)(\varkappa+2) \cdots(\varkappa+n-1), & n \in \mathbb{N} \\
1, & n=0 .\end{cases}
\end{aligned}
$$

If $f \in \mathscr{A}$ is given by (1), then it follows from (7) that

$$
\begin{array}{r}
\mathscr{L}(a, c) f(z)=z+\sum_{k=1}^{\infty} \frac{(a)_{k}}{(c)_{n}} a_{k+1} z^{k+1} \quad(z \in U), \\
z(\mathscr{L}(a, c) f)^{\prime}(z) \\
=a \mathscr{L}(a+1, c) f(z)-(a-1) \mathscr{L}(a, c) f(z) \\
(z \in \mathbb{U}) .
\end{array}
$$

We note that for $f \in \mathscr{A}$

(i) $\mathscr{L}(a, a) f(z)=f(z)$;

(ii) $\mathscr{L}(2,1) f(z)=z f^{\prime}(z)$;

(iii) $\mathscr{L}(3,1) f(z)=z f^{\prime}(z)+(1 / 2) z^{2} f^{\prime \prime}(z)$;

(iv) $\mathscr{L}(m+1,1) f(z)=\mathscr{D}^{m} f(z)=\left(z /(1-z)^{m+1}\right)$ * $f(z)(m \in \mathbb{Z} ; m>-1)$, the well-known Ruscheweyh derivative [4] of $f$;

(v) $\mathscr{L}(2,2-\mu) f(z)=\Omega_{z}^{\mu} f(z)(0 \leq \mu<1 ; z \in \mathbb{U})$, the well-known Owa-Srivastava fractional differential operator [5]. We also observe that $\Omega_{z}^{0} f(z)=f(z)$ and $\Omega_{z}^{1} f(z)=\lim _{z \rightarrow 1} \Omega_{z}^{\mu} f(z)=z f^{\prime}(z)$.

With the aid of the linear operator $\mathscr{L}(a, c)$, we introduce a subclass of $\mathscr{A}$ as follows.

Definition 1. A function $f \in \mathscr{A}$ is said to be in the class $\mathscr{R}(a, c)$, if it satisfies the condition

$$
\left|\left(\frac{\mathscr{L}(a, c) f(z)}{z}\right)^{2}-1\right|<1 \quad(z \in \mathbb{U}) .
$$

It follows from (12) and the definition of subordination that a function $f \in \mathscr{R}(a, c)$ satisfies the following subordination relation:

$$
\frac{\mathscr{L}(a, c) f(z)}{z} \prec \sqrt{1+z} \quad(z \in \mathbb{U}) .
$$

We further note that if $f \in \mathscr{R}(a, c)$, then the function $\mathscr{L}(a, c) f(z) / z$ lies in the region bounded by the right half of the lemniscate of Bernoulli given by

$$
\begin{aligned}
\left\{\omega \in \mathbb{C}:\left|\omega^{2}-1\right|<1\right\}=\{u & +i v \\
& \left.:\left(u^{2}+v^{2}\right)^{2}=2\left(u^{2}-v^{2}\right)\right\} .
\end{aligned}
$$

Noonan and Thomas [6] defined the qth Hankel determinant of a sequence $a_{k}, a_{k+1}, a_{k+2}, \ldots$ of real or complex numbers by

$$
H_{q}(k)=\left|\begin{array}{cccc}
a_{k} & a_{k+1} & \cdots & a_{k+q-1} \\
a_{k+1} & a_{k+2} & \cdots & a_{k+q} \\
\vdots & \vdots & \vdots & \vdots \\
a_{k+q-1} & a_{k+q} & \cdots & a_{k+2 q-2}
\end{array}\right| \quad(k, q \in \mathbb{N})
$$

This determinant has been studied by several authors including Noor [7] with the subject of inquiry ranging from the rate of growth of $H_{q}(k)$ (as $k \rightarrow \infty$ ) to the determination of precise bounds with specific values of $k$ and $q$ for certain subclasses of analytic functions in the unit disc $\mathbb{U}$.

For $k=1, q=2, a_{1}=1$, and $k=q=2$, the Hankel determinant simplifies to

$$
H_{2}(1)=\left|a_{3}-a_{2}^{2}\right|, \quad H_{2}(2)=\left|a_{2} a_{4}-a_{3}^{2}\right| .
$$

The Hankel determinant $H_{2}(1)$ was considered by Fekete and Szegö [8] and we refer to $H_{2}(2)$ as the second Hankel determinant. It is known [9] that if $f$ given by (1) is analytic and univalent in $\mathbb{U}$, then the sharp inequality $H_{2}(1)=\mid a_{3}-$ $a_{2}^{2} \mid \leq 1$ holds. For a family $\mathscr{F}$ of functions in $\mathscr{A}$ of the form (1), the more general problem of finding the sharp upper bounds for the functionals $\left|a_{3}-\lambda a_{2}^{2}\right|(\lambda \in \mathbb{C}$ or $\lambda \in \mathbb{R})$ is popularly known as Fekete-Szegö problem for the class $\mathscr{F}$. The Fekete-Szegö problem for various known subclasses of univalent functions (i.e., starlike, convex, close-to-convex, etc.) has been completely settled $[8,10-12]$. Recently, Janteng et al. $[13,14]$ have obtained the sharp upper bounds to the second Hankel determinant $H_{2}$ (2) for the family of functions in $\mathscr{A}$ whose derivatives have positive real part in $\mathbb{U}$. For initial work on this class of functions, one may refer to the work of MacGregor [15].

In our present investigation, we follow the techniques adopted by Libera and Złotkiewicz $[16,17]$ to solve the FeketeSzegö problem and also determine the sharp upper bound to the second Hankel determinant for the class $\mathscr{R}(a, c)$.

To establish our main results, we will need the following lemma for functions belonging to the class $\mathscr{P}$. 
Lemma 2. Let the function $\phi$, given by (4), be a member of the class $\mathscr{P}$. Then

$$
\begin{gathered}
\left|p_{k}\right| \leq 2 \quad(k \geq 1), \\
\left|p_{2}-v p_{1}^{2}\right| \leq 2 \max \{1,|2 \nu-1|\} \quad(\nu \in \mathbb{C}), \\
p_{2}=\frac{1}{2}\left\{p_{1}^{2}+\left(4-p_{1}^{2}\right) x\right\}, \\
p_{3}=\frac{1}{4}\left\{p_{1}^{3}+2\left(4-p_{1}^{2}\right) p_{1} x\right. \\
\left.-\left(4-p_{1}^{2}\right) p_{1} x^{2}+2\left(4-p_{1}^{2}\right)\left(1-|x|^{2}\right) z\right\},
\end{gathered}
$$

for some complex numbers $x, z$ satisfying $|x| \leq 1$ and $|z| \leq 1$. The estimates in (17) and (18) are sharp for the functions defined in $\mathbb{U}$ by

$$
f(z)=\frac{1+z}{1-z}, \quad g(z)=\frac{1+z^{2}}{1-z^{2}} .
$$

We note that the estimate (17) is contained in [9]; the estimate (18) is due to Ma and Minda [18], whereas the results in (19) are obtained by Libera and Złotkiewicz [17] (see also [16]).

\section{Main Results}

Unless otherwise mentioned, we assume throughout the sequel that $a \geq c>0$.

Now, we solve the Fekete-Szegö problem for the class $\mathscr{R}(a, c)$.

Theorem 3. If the function $f$, given by (1), belongs to the class $\mathscr{R}(a, c)$, then for any $\lambda \in \mathbb{C}$

$$
\left|a_{3}-\lambda a_{2}^{2}\right| \leq \frac{1}{2} \frac{(c)_{2}}{(a)_{2}} \max \left\{1, \frac{|a(c+1)+2 \lambda c(a+1)|}{4 a(c+1)}\right\} .
$$

The estimate is sharp.

Proof. From (13), it follows that

$$
\frac{\mathscr{L}(a, c) f(z)}{z}=\sqrt{1+\omega(z)} \quad(z \in \mathbb{U}),
$$

where $\omega$ is analytic and satisfies the conditions $\omega(0)=0$ and $|\omega(z)|<1$ in $\mathbb{U}$. Setting

$$
\phi(z)=\frac{1+\omega(z)}{1-\omega(z)}=1+p_{1} z+p_{2} z^{2}+\cdots \quad(z \in \mathbb{U})
$$

we see that $\phi \in \mathscr{P}$. From the above expression, we get

$$
\omega(z)=\frac{\phi(z)-1}{\phi(z)+1} \quad(z \in \mathbb{U})
$$

so that, by (22), we get

$$
\frac{\mathscr{L}(a, c) f(z)}{z}=\left(\frac{2 \phi(z)}{1+\phi(z)}\right)^{1 / 2} \quad(z \in \mathbb{U}) .
$$

Now, it is easily seen that

$$
\begin{aligned}
\left(\frac{2 \phi(z)}{1+\phi(z)}\right)^{1 / 2}= & 1+\frac{1}{4} p_{1} z+\left(\frac{1}{4} p_{2}-\frac{5}{32} p_{1}^{2}\right) z^{2} \\
& +\left(\frac{1}{4} p_{3}-\frac{5}{16} p_{1} p_{2}+\frac{13}{128} p_{1}^{3}\right) z^{3}+\cdots
\end{aligned}
$$

Differentiating the series expansion of $f$ given by (1) with respect to $z$ and comparing the coefficients of $z, z^{2}$, and $z^{3}$ in (26), we deduce that

$$
\begin{aligned}
& a_{2}=\frac{c}{4 a} p_{1} \\
& a_{3}=\frac{(c)_{2}}{(a)_{2}}\left(\frac{1}{4} p_{2}-\frac{5}{32} p_{1}^{2}\right) . \\
& a_{4}=\frac{(c)_{3}}{(a)_{3}}\left(\frac{1}{4} p_{3}-\frac{5}{16} p_{1} p_{2}+\frac{13}{128} p_{1}^{3}\right) .
\end{aligned}
$$

Thus, by using (27) and (28), we get

$$
\left|a_{3}-\lambda a_{2}^{2}\right|=\frac{1}{4} \frac{(c)_{2}}{(a)_{2}}\left|p_{2}-\frac{5 a(c+1)+2 \lambda c(a+1)}{8 a(c+1)} p_{1}^{2}\right|
$$

which with the aid of (18) yields the required estimate (21). The estimate (21) is sharp for the function $f \in \mathscr{A}$ defined in $\mathbb{U}$ by

$$
\begin{aligned}
& f(z) \\
& = \begin{cases}\varphi(c, a ; z) \star z \sqrt{1+z^{2}}, & \frac{|a(c+1)+2 \lambda c(a+1)|}{4 a(c+1)} \leq 1 \\
\varphi(c, a ; z) \star z \sqrt{1+z}, & \frac{|a(c+1)+2 \lambda c(a+1)|}{4 a(c+1)}>1 .\end{cases}
\end{aligned}
$$

Remark 4. If the function $f$, given by (1), belongs to the class $\mathscr{R}(a, c)$, then it follows at once from (27) that $\left|a_{2}\right| \leq c / 2 a$ and Theorem 3 gives $\left|a_{3}\right| \leq(c)_{2} / 2(a)_{2}$. The inequality for $\left|a_{2}\right|$ is sharp when $f$ is defined by

$$
f(z)=\varphi(c, a ; z) \star z \sqrt{1+z} \quad(z \in \mathbb{U}),
$$

and the estimate for $\left|a_{3}\right|$ is sharp for the function $g$ defined by

$$
g(z)=\varphi(c, a ; z) \star z \sqrt{1+z^{2}} \quad(z \in \mathbb{U})
$$

For the case $\lambda \in \mathbb{R}$, Theorem 3 reduces to the following result. 
Corollary 5. Let $\lambda \in \mathbb{R}$. If the function $f$, given by (1), belongs to the class $\mathscr{R}(a, c)$, then

$$
\begin{aligned}
& \left|a_{3}-\lambda a_{2}^{2}\right| \\
& \leq\left\{\begin{array}{lc}
-\frac{c\{a(c+1)+2 \lambda(a+1) c\}}{8 a(a)_{2}}, & \lambda<-\frac{5 a(c+1)}{2(a+1) c} \\
\frac{(c)_{2}}{2(a)_{2}}, & -\frac{5 a(c+1)}{2(a+1) c} \\
\frac{c\{a(c+1)+2 \lambda c(a+1)\}}{8 a(a)_{2}}, & \lambda>\frac{3 a(c+1)}{2(a+1) c} .
\end{array}\right.
\end{aligned}
$$

The estimate is sharp for the function $f$ defined in $\mathbb{U}$ by

$$
\begin{aligned}
& f(z) \\
& =\left\{\begin{array}{rr}
\varphi(c, a ; z) \star z \sqrt{1+z}, & \lambda<-\frac{5 a(c+1)}{2(a+1) c} \\
& \text { or } \lambda>\frac{3 a(c+1)}{2(a+1) c} \\
\varphi(c, a ; z) \star z \sqrt{1+z^{2}}, & -\frac{5 a(c+1)}{2(a+1) c} \\
\leq \lambda \leq \frac{3 a(c+1)}{2(a+1) c} .
\end{array}\right.
\end{aligned}
$$

Putting $a=2$ and $c=1$ in Corollary 5, we get the following.

Corollary 6. If the function $f$, given by (1), satisfies the subordination relation

$$
f^{\prime}(z) \prec \sqrt{1+z} \quad(z \in \mathbb{U}),
$$

then

$$
\left|a_{3}-\lambda a_{2}^{2}\right| \leq \begin{cases}-\frac{2+3 \lambda}{48}, & \lambda<-\frac{10}{3} \\ \frac{1}{6}, & -\frac{10}{3} \leq \lambda \leq 2 \\ \frac{2+3 \lambda}{48}, & \lambda>2 .\end{cases}
$$

The estimate is sharp for the function $f$ defined in $\mathbb{U}$ by

$$
f(z)= \begin{cases}\int_{0}^{z} \frac{d t}{1-t} \star z \sqrt{1+z}, & \lambda<-\frac{10}{3} \text { or } \lambda>2 \\ \int_{0}^{z} \frac{d t}{1-t} \star z \sqrt{1+z^{2}}, & -\frac{10}{3} \leq \lambda \leq 2 .\end{cases}
$$

In the following theorem, we find the sharp upper bound to the second Hankel determinant for the class $\mathscr{R}(a, c)$.

Theorem 7. If $a \geq c \geq 1 / 2$ and the function $f$, given by (1), belongs to the class $\mathscr{R}(a, c)$, then

$$
\left|a_{2} a_{4}-a_{3}^{2}\right| \leq\left\{\frac{(c)_{2}}{2(a)_{2}}\right\}^{2} .
$$

The estimate in (39) is sharp for the function g, given by (33).
Proof. From (27), (28), and (29), we deduce that

$$
\begin{aligned}
\left|a_{2} a_{4}-a_{3}^{2}\right|=\frac{1}{16} \mid & \mid \frac{c}{a} \frac{(c)_{3}}{(a)_{3}}\left(p_{1} p_{3}-\frac{5}{4} p_{1}^{2} p_{2}+\frac{13}{32} p_{1}^{4}\right) \\
& -\left\{\frac{(c)_{2}}{(a)_{2}}\right\}^{2}\left(p_{2}^{2}-\frac{5}{4} p_{1}^{2} p_{2}+\frac{25}{64} p_{1}^{4}\right) \mid .
\end{aligned}
$$

Since the functions $\phi(z)$ and $\phi\left(e^{i \theta} z\right)(\theta \in \mathbb{R})$ are in the class $\mathscr{P}$ simultaneously, we assume without loss of generality that $p_{1}>0$. For convenience of notation, we write $p_{1}=p(0 \leq$ $p \leq 2)$. Now, by using (19) in (40), we get

$$
\begin{aligned}
&\left|a_{2} a_{4}-a_{3}^{2}\right|=\frac{1}{16} \mid \frac{c}{4 a} \frac{(c)_{3}}{(a)_{3}}\left\{p^{4}+2\left(4-p^{2}\right) p^{2} x\right.-\left(4-p^{2}\right) p^{2} x^{2} \\
&\left.\quad+2\left(4-p^{2}\right)\left(1-|x|^{2}\right) p z\right\} \\
&- \frac{5(a-c) c(c)_{2}}{8(a)_{2}(a)_{3}}\left\{p^{4}+\left(4-p^{2}\right) p^{2} x\right\} \\
&-\frac{1}{4}\left\{\frac{(c)_{2}}{(a)_{2}}\right\}^{2} \\
& \times\left\{p^{4}+\left(4-p^{2}\right)^{2} x^{2}+2 p^{2}\left(4-p^{2}\right) x\right\} \\
&+\frac{(a-c) c(c)_{2}}{64(a)_{2}(a)_{3}} p^{4} \mid
\end{aligned}
$$

for some $x(|x| \leq 1)$ and for some $z(|z| \leq 1)$. Applying the triangle inequality in (41) and replacing $|x|$ by $y$ in the resulting equation, we get

$$
\begin{aligned}
& \left|a_{2} a_{4}-a_{3}^{2}\right| \\
& \leq \frac{c(c)_{2}}{32 a(a)_{2}} \\
& \times\left\{\frac{a c+3 a+2}{32(a+1)_{2}} p^{4}\right. \\
& +\frac{a-c}{4(a+1)_{2}}\left(4-p^{2}\right) p^{2} y \\
& +\frac{(2-p)\left(4-p^{2}\right)\{2(a+2)(c+1)-(a-c) p\}}{2(a+1)_{2}} y^{2} \\
& \left.+\frac{c+2}{a+2}\left(4-p^{2}\right) p\right\} \\
& =\mathscr{G}(p, y) \quad(0 \leq p \leq 2,0 \leq y \leq 1) \quad \text { (say). }
\end{aligned}
$$


We next maximize the function $\mathscr{G}(p, y)$ on the closed rectangle $[0,2] \times[0,1]$. Since

$$
\begin{aligned}
\frac{\partial \mathscr{G}}{\partial y} & \\
= & \frac{c(c)_{2}}{32 a(a)_{2}} \\
& \times\left\{\frac{a-c}{4(a+1)_{2}}\left(4-p^{2}\right) p^{2}\right. \\
& \left.\quad+\frac{(2-p)\left(4-p^{2}\right)\{(a+2)(c+1)-(a-c) p\}}{2(a+1)_{2}} y\right\}
\end{aligned}
$$$$
>0
$$

for $0<p<2$ and $0<y<1$, the function $\mathscr{G}(p, y)$ cannot have a maximum value in the interior on the closed rectangle $[0,2] \times[0,1]$. Therefore, for fixed $p \in[0,2]$,

$$
\max _{0 \leq y \leq 1} \mathscr{G}(p, y)=\mathscr{G}(p, 1)=\mathscr{F}(p) \quad \text { (say) }
$$

where

$$
\begin{aligned}
\mathscr{F}(p)= & \frac{c(c)_{2}}{32 a(a)_{2}} \\
& \times\left\{\frac{a c-21 a+24 c+2}{32(a+1)_{2}} p^{4}\right. \\
& +\frac{a-7 c-2 a c-4}{(a+1)_{2}} p^{2} \\
& \left.+\frac{8(c+1)}{a+1}\right\} \quad(0 \leq p \leq 2) .
\end{aligned}
$$

Setting

$$
\begin{aligned}
& \mathscr{F}^{\prime}(p) \\
&= \frac{c(c)_{2}}{256 a(a)_{2}(a+1)_{2}} \\
& \quad \times\left\{(a c-21 a+24 c+2) p^{2}\right. \\
&\quad+16(a-7 c-2 a c-4)\} p=0,
\end{aligned}
$$

we note that either $p=0$ or

$$
p^{2}=\frac{16(2 a c+7 c-a+4)}{a c-21 a+24 c+2}>4 .
$$

Since $a<2 a c+7 c+4$, we further observe that $\mathscr{F}^{\prime \prime}(0)<0$. Thus, the maximum value of $\mathscr{F}$ is attained at $p=0$ so that the upper bound in (42) corresponds to $p=0$ and $y=1$ from which we get the assertion of the theorem.

Letting $a=2$ and $c=1$ in Theorem 7 , we get the following.
Corollary 8. If the function $f$, given by (1), satisfies the condition (36), then

$$
\left|a_{2} a_{4}-a_{3}^{2}\right| \leq \frac{1}{36} .
$$

and the estimate is sharp for the function $f$ defined by

$$
f(z)=\int_{0}^{z} \frac{d t}{1-t} \star z \sqrt{1+z^{2}} \quad(z \in \mathbb{U}) .
$$

Next, we find the sharp upper bound for the fourth coefficient of functions belonging to the class $\mathscr{R}(a, c)$.

Theorem 9. If the function $f$, given by (1), belongs to the class $\mathscr{R}(a, c)$, then

$$
\left|a_{4}\right| \leq \frac{1}{2} \frac{(c)_{3}}{(a)_{3}}
$$

The estimate is sharp.

Proof. Using (19) in (29) and following the lines of proof of Theorem 7, we deduce that

$$
\begin{aligned}
\left|a_{4}\right|= & \frac{(c)_{3}}{(a)_{3}} \\
& \times \mid \frac{p^{3}}{128}-\frac{\left(4-p^{2}\right) p}{32} x \\
& \quad-\frac{\left(4-p^{2}\right) p}{16} x^{2}+\frac{\left(4-p^{2}\right)}{8}\left(1-|x|^{2}\right) z \mid
\end{aligned}
$$

for some $(|x| \leq 1)$ and $z(|z| \leq 1)$. By an application of the triangle inequality in the above expression followed by replacement of $|x|$ by $y$ in the resulting equation, we obtain

$$
\begin{aligned}
\left|a_{4}\right| \leq & \frac{(c)_{3}}{(a)_{3}} \\
& \times\left\{\frac{p^{3}}{128}+\frac{\left(4-p^{2}\right) p}{32} y\right. \\
& \left.\quad-\frac{\left(4-p^{2}\right)(2-p)}{16} y^{2}+\frac{\left(4-p^{2}\right)}{8}\right\} \\
= & G(p, y) \quad(0 \leq p \leq 2,0 \leq y \leq 1) \text { (say). }
\end{aligned}
$$

We next maximize the function $G(p, y)$ on the closed rectangle $[0,2] \times[0,1]$. Since

$$
\frac{\partial G}{\partial y}=\frac{(c)_{3}}{32(a)_{3}}\left(4-p^{2}\right)\{p-4(2-p) y\}<0
$$

for $0<p<2$ and $0<y<1$, it follows that $G(p, y)$ cannot have a maximum value in the interior of the closed rectangle $[0,2] \times[0,1]$. Thus, for fixed $p \in[0,2]$,

$$
\max _{0 \leq y \leq 1} G(p, y)=G(p, 0)=F(p) \quad(\text { say })
$$


where

$$
F(p)=\frac{(c)_{3}}{128(a)_{3}}\left(p^{3}-16 p^{2}+64\right)
$$

We further note that

$$
F^{\prime}(p)=\frac{(c)_{3}}{128(a)_{3}}(3 p-32) p=0
$$

for $p=0$ or $p=32 / 3$. Since $F^{\prime \prime}(0)=-(c)_{3} / 4(a)_{3}<0$, the function $F$ attains its maximum value at $p=0$. Thus, the upper bound of the function $G$ corresponds to $p=y=0$. Putting $p=y=0$ in (52), we get our desired estimate (50).

The estimate in (50) is sharp for the function $f$ defined by

$$
f(z)=\varphi(c, a ; z) \star z \sqrt{1+z^{3}} \quad(z \in \mathbb{U}) .
$$

Letting $a=2$ and $c=1$ in Theorem 9, we obtain the following.

Corollary 10. If the function $f$, given by (1), satisfies the condition (36), then

$$
\left|a_{4}\right| \leq \frac{1}{8}
$$

and the estimate is sharp for the function $f$ defined by

$$
f(z)=\int_{0}^{z} \frac{d t}{1-t} \star z \sqrt{1+z^{3}} \quad(z \in \mathbb{U}) .
$$

Finally, we obtain a sufficient condition for a function in $\mathscr{A}$ to be in the class $\mathscr{R}(a, c)$.

Theorem 11. Let $\gamma>0$. If $f \in \mathscr{A}$ satisfies

$$
\operatorname{Re}\left\{\frac{\mathscr{L}(a+1, c) f(z)}{\mathscr{L}(a, c) f(z)}\right\}<1+\frac{1}{2 a \gamma} \quad(z \in \mathbb{U}),
$$

then

$$
\frac{\mathscr{L}(a, c) f(z)}{z} \prec(1+z)^{1 / \gamma} \quad(z \in \mathbb{U})
$$

and the result is the best possible.

Proof. Setting

$$
\frac{\mathscr{L}(a, c) f(z)}{z}=(1+w(z))^{1 / \gamma} \quad(z \in \mathbb{U})
$$

and choosing the principal branch in (62), we see that $w$ is analytic in $\mathbb{U}$ with $w(0)=0$. Taking the logarithmic differentiation in (62) and using the identity (11) in the resulting equation, we deduce that

$$
\frac{\mathscr{L}(a+1, c) f(z)}{\mathscr{L}(a, c) f(z)}=1+\frac{1}{a \gamma} \frac{z w^{\prime}(z)}{1+w(z)} \quad(z \in \mathbb{U}) .
$$

We claim that $|w(z)|<1$ for all $z \in \mathbb{U}$. Otherwise, there exists a point $z_{0} \in \mathbb{U}$ such that

$$
\max _{|z| \leq\left|z_{0}\right|}|w(z)|=\left|w\left(z_{0}\right)\right|=1 \quad\left(w\left(z_{0}\right) \neq 1\right) .
$$

Letting $w\left(z_{0}\right)=e^{i \theta}(-\pi<\theta \leq \pi)$ and applying Jack's lemma [19], we have

$$
z_{0} w^{\prime}\left(z_{0}\right)=k w\left(z_{0}\right) \quad(k \geq 1) .
$$

Using (65) in (63), we get

$$
\begin{aligned}
\operatorname{Re}\left\{\frac{\mathscr{L}(a+1, c) f(z)}{\mathscr{L}(a, c) f(z)}\right\} & =1+\frac{1}{a \gamma} \operatorname{Re}\left\{\frac{z_{0} w^{\prime}\left(z_{0}\right)}{1+w\left(z_{0}\right)}\right\} \\
& =1+\frac{k}{a \gamma} \operatorname{Re}\left\{\frac{e^{i \theta}}{1+e^{i \theta}}\right\} \\
& \geq 1+\frac{1}{2 a \gamma},
\end{aligned}
$$

which contradicts the hypothesis (60). Thus, we conclude that $|w(z)|<1$ for all $z \in \mathbb{U}$ and the assertion of the theorem follows from (62).

To see that the result is the best possible, we consider the principal branch of the function $f_{0}$ defined by

$$
f_{0}(z)=\varphi(z) \star z(1+z)^{1 / \gamma} \quad(z \in \mathbb{U}) .
$$

Then, it follows from (67) that

$$
\frac{\mathscr{L}(a, c) f_{0}(z)}{z}=(1+z)^{1 / \gamma} \quad(z \in \mathbb{U}) .
$$

On differentiating the above expression logarithmically followed by the use of the identity (11), we obtain

$$
\begin{aligned}
\frac{\mathscr{L}(a+1, c) f_{0}(z)}{\mathscr{L}(a, c) f_{0}(z)} & =1+\frac{1}{a \gamma} \frac{z}{1+z} \\
& \longrightarrow 1+\frac{1}{2 a \gamma} \text { as } z \longrightarrow 1^{-}
\end{aligned}
$$

The proof of the theorem is thus completed.

For $\gamma=2$ in Theorem 11, we have the following.

Corollary 12. If $f \in \mathscr{A}$ satisfies

$$
\operatorname{Re}\left\{\frac{\mathscr{L}(a+1, c) f(z)}{\mathscr{L}(a, c) f(z)}\right\}<1+\frac{1}{4 a} \quad(z \in \mathbb{U}),
$$

then $f \in \mathscr{R}(a, c)$. The result is the best possible.

Remark 13. Further, by specializing the parameters $a$ and $c$, one can obtain interesting subclasses of $\mathscr{A}$ involving the various operators discussed in the introduction and the corresponding results obtained here can be extended to these classes. 


\section{Conflict of Interests}

The authors declare that there is no conflict of interests regarding the publication of this paper.

\section{References}

[1] S. S. Miller and P. T. Mocanu, "Differential subordinations and univalent functions," The Michigan Mathematical Journal, vol. 28, no. 2, pp. 157-172, 1981.

[2] S. S. Miller and P. T. Mocanu, Differential Subordinations: Theory and Applications, vol. 225 of Monographs and Textbooks in Pure and Applied Mathematics, Marcel Dekker, New York, NY, USA, 2000.

[3] B. C. Carlson and D. B. Shaffer, "Starlike and prestarlike hypergeometric functions," SIAM Journal on Mathematical Analysis, vol. 15, no. 4, pp. 737-745, 1984.

[4] S. Ruscheweyh, "New criteria for univalent functions," Proceedings of the American Mathematical Society, vol. 49, pp. 109-115, 1975.

[5] S. Owa and H. M. Srivastava, "Univalent and starlike generalized hypergeometric functions," Canadian Journal of Mathematics, vol. 39, no. 5, pp. 1057-1077, 1987.

[6] J. W. Noonan and D. K. Thomas, "On the second Hankel determinant of areally mean $p$-valent functions," Transactions of the American Mathematical Society, vol. 223, pp. 337-346, 1976.

[7] K. I. Noor, "Hankel determinant problem for the class of functions with bounded boundary rotation," Revue Roumaine de Mathématique Pures et Appliquées, vol. 28, no. 8, pp. 731-739, 1983.

[8] M. Fekete and G. Szegö, "Eine bemerkung über ungerade schlichte funktionen," Journal of the London Mathematical Society, vol. 8, pp. 85-89, 1933.

[9] P. L. Duren, Univalent Functions, Grundlehren der Mathematischen Wissenschaften, Springer, New York, NY, USA, 1983.

[10] F. R. Keogh and E. P. Merkes, "A coefficient inequality for certain classes of analytic functions.", Proceedings of the American Mathematical Society, vol. 20, pp. 8-12, 1969.

[11] W. Koepf, "On the Fekete-Szegö problem for close-to-convex functions II," Archiv der Mathematik, vol. 49, no. 5, pp. 420-433, 1987.

[12] W. Koepf, "On the Fekete-Szegö problem for close-to-convex functions," Proceedings of the American Mathematical Society, vol. 101, no. 1, pp. 89-95, 1987.

[13] A. Janteng, S. A. Halim, and M. Darus, "Coefficient inequality for a function whose derivative has a positive real part," Journal of Inequalities in Pure and Applied Mathematics, vol. 7, no. 2, article 50, 2006.

[14] A. Janteng, S. A. Halim, and M. Darus, "Estimate on the second Hankel functional for functions whose derivative has a positive real part," Journal of Quality Measurement and Analysis, vol. 4, pp. 189-195, 2008.

[15] T. H. MacGregor, "Functions whose derivative has a positive real part," Transactions of the American Mathematical Society, vol. 104, pp. 532-537, 1962.

[16] R. J. Libera and E. J. Złotkiewicz, "Early coefficients of the inverse of a regular convex function," Proceedings of the American Mathematical Society, vol. 85, no. 2, pp. 225-230, 1982.

[17] R. J. Libera and E. J. Złotkiewicz, "Coefficient bounds for the inverse of a function with derivative in $\mathscr{P}$," Proceedings of the American Mathematical Society, vol. 87, no. 2, pp. 251-257, 1983.
[18] W. C. Ma and D. Minda, "A unified treatment of some special classes of univalent functions," in Proceedings of the Conference on Complex Analysis (Tianjin, 1992), Z. Li, F. Ren, L. Yang and, and S. Zhang, Eds., pp. 157-169, International Press, Cambridge, Mass, USA, 1994.

[19] I. S. Jack, "Functions starlike and convex of order $\alpha$," Journal of the London Mathematical Society, vol. 3, pp. 469-474, 1971. 


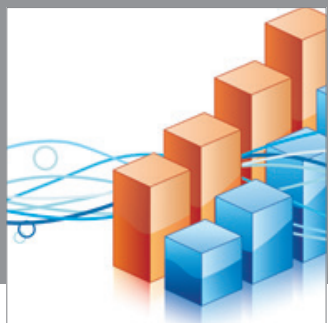

Advances in

Operations Research

mansans

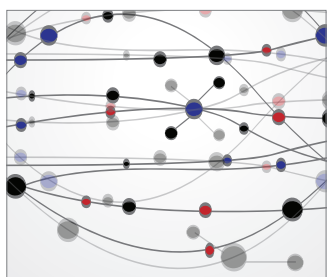

The Scientific World Journal
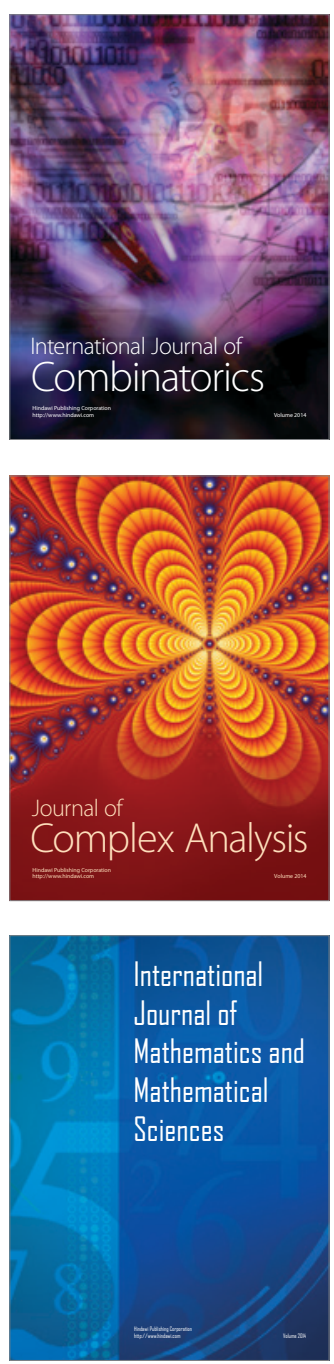
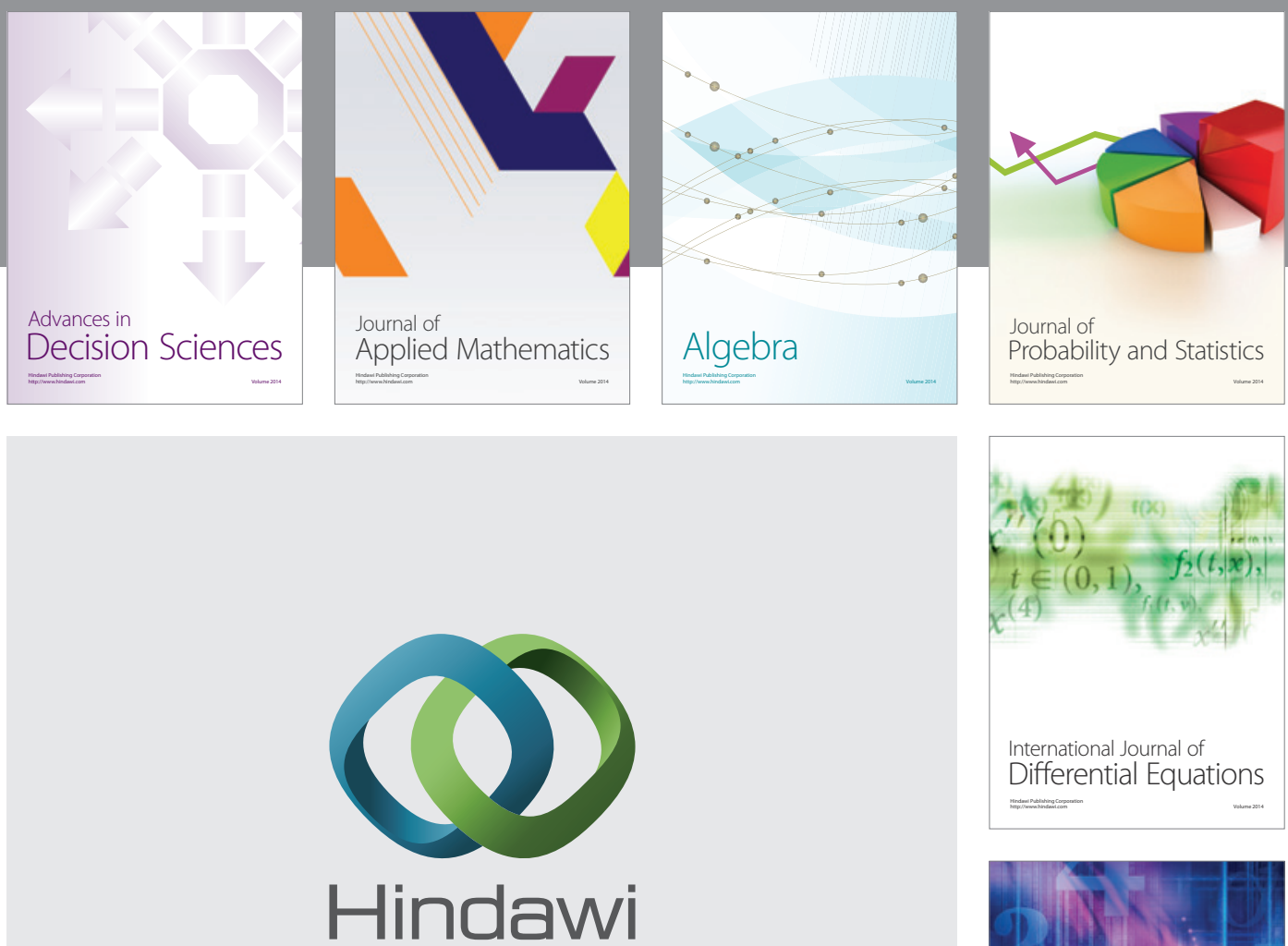

Submit your manuscripts at http://www.hindawi.com
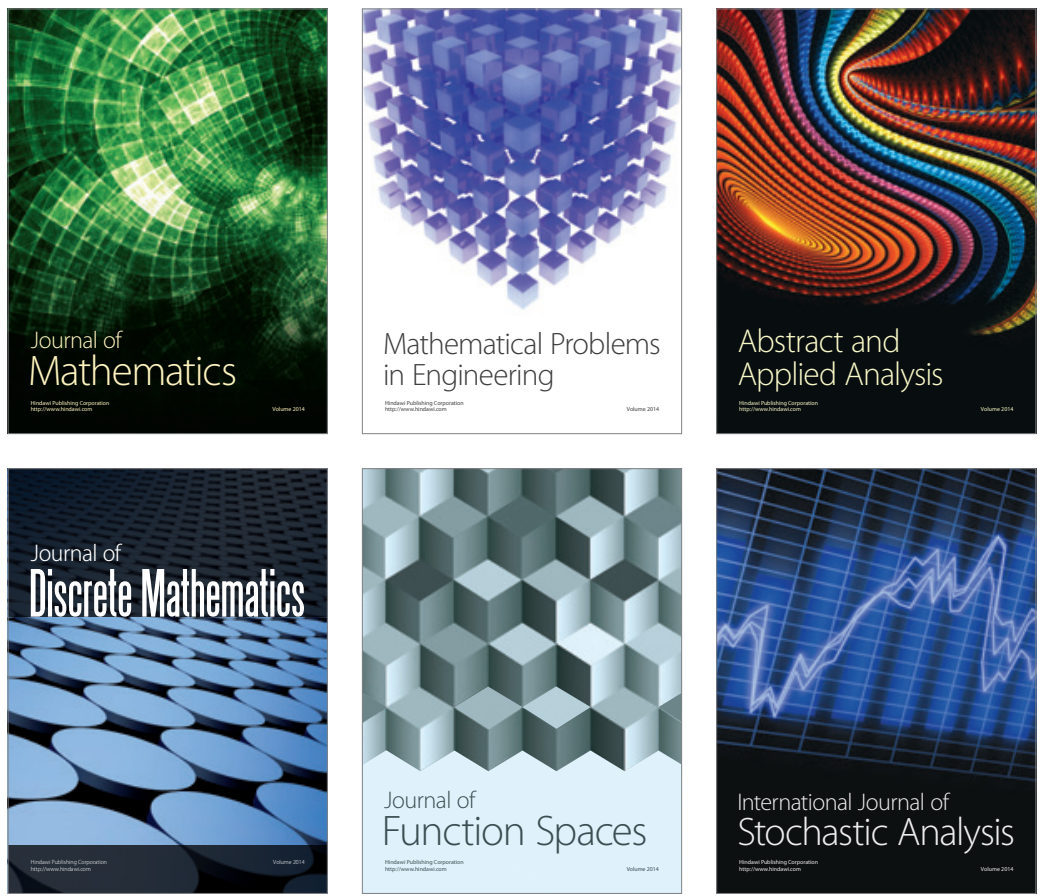

Journal of

Function Spaces

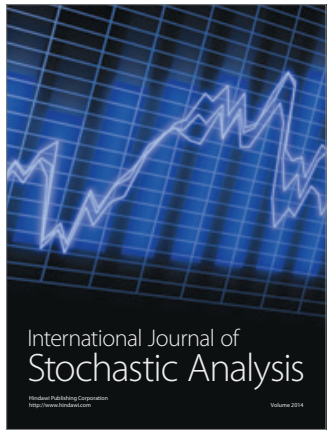

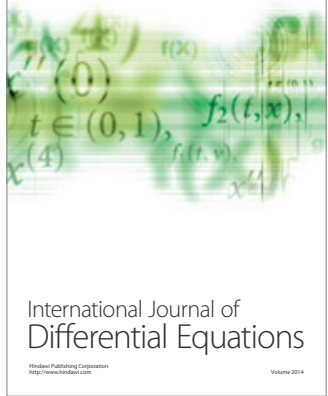
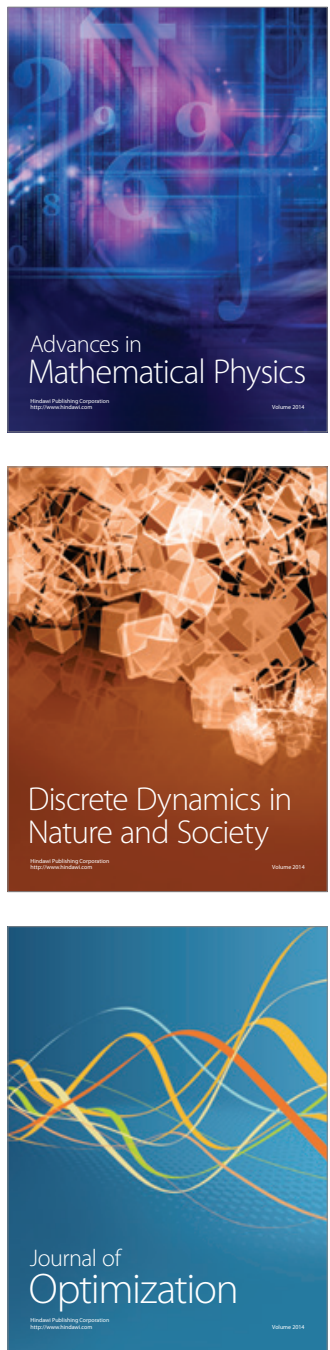